\title{
Subharmonic solutions for a class of second-order impulsive Lagrangian systems with damped term
}

\section{Xingyong Zhang*}

"Correspondence:
zhangxingyong1@163.com
Department of Mathematics,
Faculty of Science, Kunming
University of Science and
Technology, Kunming, Yunnan
650500, P.R. China

\begin{abstract}
In this paper, by using the mountain pass theorem, we investigate the existence of subharmonic weak solutions for a class of second-order impulsive Lagrangian systems with damped term under asymptotically quadratic conditions. Some new existence criteria are established. Finally, an example is presented to verify our results.
\end{abstract} MSC: $37 \mathrm{~J} 45 ; 34 \mathrm{C} 25 ; 70 \mathrm{H} 05$

Keywords: impulsive Lagrangian systems; damped term; subharmonic weak solutions; mountain pass theorem

\section{Introduction and main results}

In this paper, we investigate the existence of subharmonic weak solutions for the following second-order impulsive Lagrangian system with damped term:

$$
\left\{\begin{array}{l}
\frac{d(P(t) \dot{u}(t))}{d t}+(q(t) P(t)+B) \dot{u}(t)+\left(\frac{1}{2} q(t) B-A(t)\right) u(t)+\nabla F(t, u(t)) \\
\quad=0, \quad \text { a.e. } t \in \mathbb{R}, \\
\Delta\left(P\left(t_{j}\right) \dot{u}\left(t_{j}\right)\right)=P\left(t_{j}\right) \dot{u}\left(t_{j}^{+}\right)-P\left(t_{j}\right) \dot{u}\left(t_{j}^{-}\right)=\nabla I_{j}\left(u\left(t_{j}\right)\right), \quad j=1, \ldots, p,
\end{array}\right.
$$

where $T>0, p \in \mathbb{N}, t_{0}=0<t_{1}<t_{2}<\cdots<t_{p}<t_{p+1}=T, u(t)=\left(u^{1}(t), \ldots, u^{N}(t)\right), I_{j}: \mathbb{R}^{N} \rightarrow \mathbb{R}$, $q \in C(\mathbb{R}, \mathbb{R})$ satisfying $q(t+T)=q(t)$ and $\int_{0}^{T} q(t) d t=0, B$ is a skew-symmetric $N \times N$ constant matrix, $P(t)$ and $A(t)$ are symmetric and continuous $N \times N$ matrix-value functions on $\mathbb{R}$ satisfying $P(t+T)=P(t)$ and $A(t+T)=A(t)$, and $F: \mathbb{R} \times \mathbb{R}^{N} \rightarrow \mathbb{R}$ satisfies $F(t, x)=-K(t, x)+W(t, x)$, where $K, W$ are $T$-periodic in their first variable, and the following assumption:

(A) $F(t, x)$ is measurable in $t$ for every $x \in \mathbb{R}^{N}$ and continuously differentiable in $x$ for a.e. $t \in[0, T]$, and there exist $a \in C\left(\mathbb{R}^{+}, \mathbb{R}^{+}\right)$and $b \in L^{1}\left(0, T ; \mathbb{R}^{+}\right)$with $b(t+T)=b(t)$ such that

$$
\begin{aligned}
& |F(t, x)| \leq a(|x|) b(t), \quad|\nabla F(t, x)| \leq a(|x|) b(t), \\
& \left|I_{j}(x)\right| \leq a(|x|), \quad\left|\nabla I_{j}(x)\right| \leq a(|x|)
\end{aligned}
$$

for all $x \in \mathbb{R}^{N}$ and a.e. $t \in[0, T]$. 
Lagrangian systems are applied extensively to study the fluid mechanics, nuclear physics and relativistic mechanics. Especially, as a special case of Lagrangian systems, the following second-order Hamiltonian systems are considered by many authors:

$$
\ddot{u}(t)=\nabla F(t, u(t)), \quad \text { a.e. } t \in \mathbb{R},
$$

where $F: \mathbb{R} \times \mathbb{R}^{N} \rightarrow \mathbb{R}$ satisfies $F(t+T, x)=F(t, x)$, and the existence and multiplicity of periodic solutions, subharmonic solutions and homoclinic solutions are obtained via variational methods. We refer readers to [1-14]. Especially, in 2010, under the asymptotically quadratic conditions, Tang and Jiang [10] obtained the following interesting result.

Theorem A (see [10], Theorem 1.1) Assume that $F$ satisfies

(F) $F(t, x)=-K(t, x)+W(t, x)$ and $K, W \in C^{1}\left(\mathbb{R} \times \mathbb{R}^{N}, \mathbb{R}\right)$ are $T$-periodic in their first variable with $T>0$, and that $K$ and $W$ satisfy the following assumptions:

(H1) There exist constants $b>0$ and $\gamma \in(1,2]$ such that

$$
K(t, 0)=0, \quad K(t, x) \geq b|x|^{\gamma} \quad \text { for }(t, x) \in[0, T] \times \mathbb{R}^{N} ;
$$

(H2) $(\nabla K(t, x), x) \leq 2 K(t, x)$ for $(t, x) \in[0, T] \times \mathbb{R}^{N}$;

(H3) $\lim \sup _{|x| \rightarrow 0} \frac{W(t, x)}{|x|^{2}}<b$ uniformly for $t \in[0, T]$;

(H4) There exists a function $g \in L^{1}([0, T], \mathbb{R})$ such that

$$
(\nabla W(t, x), x)-2 W(t, x) \geq g(t) \quad \text { for }(t, x) \in[0, T] \times \mathbb{R}^{N}
$$

and

$$
\lim _{|x| \rightarrow \infty}[(\nabla W(t, x), x)-2 W(t, x)]=+\infty \quad \text { for a.e. } t \in[0, T]
$$

(H5) There exist constants $a>0$ and $d>0$ such that

$$
W(t, x) \leq a|x|^{2}+d \quad \text { for }(t, x) \in[0, T] \times \mathbb{R}^{N} ;
$$

(H6) There exists $x_{0} \in \mathbb{R}^{N}$ such that

$$
\int_{0}^{T}\left[K\left(t, x_{0}\right)-W\left(t, x_{0}\right)-\frac{g(t)}{2}\right] d t<0
$$

Then system (1.2) has a nontrivial T-periodic solution.

In recent years, variational methods have been applied to study the existence and multiplicity of solutions for impulsive differential equations and lots of interesting results have been obtained, see [15-20].

In [15], Nieto and O'Regan considered a one-dimensional Dirichlet boundary value problem with impulses. They obtained that the solutions of the impulsive problem minimize some (energy) functional and the critical points of the functional are indeed solutions of the impulsive problem. 
In [16], Nieto introduced a variational formulation for the following one-dimensional damped nonlinear Dirichlet problem with impulses:

$$
\left\{\begin{array}{l}
u^{\prime \prime}(t)+g(t) u^{\prime}(t)+\lambda u(t)=f(t, u(t)), \quad t \in[0, T] \\
\Delta u^{\prime}\left(t_{j}\right)=I_{j}\left(u\left(t_{j}^{-}\right)\right), \quad j=1,2, \ldots, p \\
u(0)=u(T)=0
\end{array}\right.
$$

and gave the concept of a weak solution for such a problem. They obtained that the weak solutions of problem (1.3) are indeed the critical points of the functional:

$$
\begin{aligned}
\varphi(v)= & \frac{1}{2} \int_{0}^{T} e^{G(t)}\left(v^{\prime}(t)\right)^{2} d t+\frac{\lambda}{2} \int_{0}^{T} e^{G(t)} v^{2}(t) d t \\
& +\sum_{j=1}^{p} e^{G\left(t_{j}\right)} \int_{0}^{v\left(t_{j}\right)} I_{j}(t) d t-\int_{0}^{T} e^{G(t)} F(t, v(t)) d t
\end{aligned}
$$

where $G(t)=\int_{0}^{t} g(t) d t$ and $F(t, v)=\int_{0}^{v} f(t, s) d s$. In [17] and [18], the authors also dealt with some one-dimensional impulsive problems with damped term by variational methods.

For higher dimensional dynamical systems, some interesting results have also been obtained (see [21-23]). In [21], Zhou and Li investigated the second-order Hamiltonian system with impulsive effects:

$$
\left\{\begin{array}{l}
\ddot{u}(t)=\nabla F(t, u(t)), \quad \text { a.e. } t \in[0, T], \\
u(0)-u(T)=\dot{u}(0)-\dot{u}(T)=0, \\
\Delta \dot{u}^{i}\left(t_{j}\right)=\dot{u}^{i}\left(t_{j}^{+}\right)-\dot{u}^{i}\left(t_{j}^{-}\right)=I_{i j}\left(u^{i}\left(t_{j}\right)\right), \quad i=1,2, \ldots, N, j=1,2, \ldots, p .
\end{array}\right.
$$

By using the least action principle and the saddle point theorem, they obtained some existence results of solutions under sublinear condition and some reasonable conditions. In [22], system (1.5) with $F(t, u)=\frac{1}{2} A(t) u \cdot u-\lambda W(t, u)-\mu G(t, u)$, where $\lambda, \mu \in \mathbb{R}$, was also investigated. By using variational methods, the authors obtained that system (1.5) has at least three weak solutions. In [23], the authors investigated system (1.5) with $F(t, u)=\frac{1}{2} A(t) u \cdot u-W(t, u)$. They obtained that system (1.5) has infinitely many solutions under the assumptions that nonlinear term is superquadratic, asymptotically quadratic and subquadratic, respectively.

In recent years, via variational methods, some authors have been interested in studying the existence and multiplicity of periodic solutions and homoclinic solutions for the following Lagrangian systems with damped term:

$$
\frac{d(P(t) \dot{u}(t))}{d t}+B \dot{u}(t)+\nabla F(t, u(t))=0,
$$

where $P(t)$ is a symmetric and continuous $N \times N$ matrix-valued function, $B$ is a skewsymmetric $N \times N$ constant matrix and $F: \mathbb{R} \times \mathbb{R}^{N} \rightarrow \mathbb{R}$. They obtained some interesting results. We refer readers to [24-27]. 
In 2010, Li et al. [28] investigated the following system, more general than system (1.6), with $P(t) \equiv I_{N}$ :

$$
\left\{\begin{array}{l}
\ddot{u}(t)+\left(q(t) I_{N \times N}+B\right) \dot{u}(t)+\left(\frac{1}{2} q(t) B-A(t)\right) u(t)+\nabla F(t, u(t)) \\
\quad=0, \quad \text { a.e. } t \in[0, T] \\
u(0)-u(T)=\dot{u}(0)-\dot{u}(T)=0 .
\end{array}\right.
$$

Motivated by [28], in [29], we investigated the following system, more general than system (1.7):

$$
\left\{\begin{array}{l}
\frac{d(P(t) \dot{u}(t))}{d t}+(q(t) P(t)+B) \dot{u}(t)+\left(\frac{1}{2} q(t) B-A(t)\right) u(t)+\nabla F(t, u(t)) \\
\quad=0, \quad \text { a.e. } t \in[0, T] \\
u(0)-u(T)=P(0) \dot{u}(0)-P(T) \dot{u}(T)=0 .
\end{array}\right.
$$

By variational methods, under superquadratic or subquadratic conditions, we obtained that system (1.8) has infinitely many solutions. One can see more details of our results and more research background of system (1.8) in [29].

In [32], Luo et al. investigated the existence of subharmonic solutions with prescribed minimal period for the following one-dimensional second-order impulsive differential equation:

$$
\begin{cases}u^{\prime \prime}(t)+f(t, u(t))=0, & \text { a.e. } t \in J^{\prime}, \\ \Delta u^{\prime}\left(t_{m}\right)=I_{m}\left(u\left(t_{m}\right)\right), & m \in Z_{0},\end{cases}
$$

where $f \in C(\mathbb{R} \times \mathbb{R}, \mathbb{R}), Z_{0}=Z^{+} \cup Z^{-}, J^{\prime}=\mathbb{R} \backslash\left\{t_{m} \mid m \in Z_{0}\right\}, I_{m} \in C\left(\mathbb{R}, \mathbb{R}^{+} \cup\{0\}\right), 0<t_{1}<$ $t_{2}<\cdots<t_{p}<T, I_{m+p}=I_{m}$ and $t_{m}=t_{m+p}-T$ if $m \in Z^{+}$, while $t_{m}=t_{m+p+1}-T$ if $m \in Z^{-}$.

In this paper, motivated by $[10,15,16,21,28,29]$ and [32], we focus on the existence of subharmonic weak solutions for system (1.1), which is of impulsive conditions, and we study the problem under asymptotically quadratic conditions. To the best of our knowledge, there are few papers that consider such a problem for system (1.1). We call a solution $u$ subharmonic if $u$ is $k T$-periodic for some $k \in \mathbb{N}$.

Let

$$
\begin{aligned}
\|A\| & =\sup _{t \in[0, T]} \max _{|x|=1, x \in \mathbb{R}^{N}}|A(t) x| \\
& =\sup _{t \in[0, T]} \max \left\{\sqrt{\lambda(t)}: \lambda(t) \text { is the eigenvalue of } A^{\tau}(t) A(t)\right\}
\end{aligned}
$$

and

$$
\begin{aligned}
\|B\| & =\max _{|x|=1, x \in \mathbb{R}^{N}}|B x| \\
& =\max \left\{\sqrt{\lambda}: \lambda \text { is the eigenvalue of } B^{\tau} B\right\} .
\end{aligned}
$$

In this paper, we make the following assumptions: 
(P) There exists a constant $m>\frac{1}{2}$ such that the matrix $P(t)$ satisfies

$$
P(0)=P(T), \quad(P(t) x, x)>m(x, x) \quad \text { for all }(t, x) \in \mathbb{R} \times\left\{\mathbb{R}^{N} /\{0\}\right\}
$$

(K1) There exist constants $a>0$ and $\gamma \in(0,2]$ such that

$$
K(t, 0)=0, \quad K(t, x) \geq\left(\frac{\|B\|^{2}+2\|A\|}{4}+a\right)|x|^{\gamma} \quad \text { for all } x \in \mathbb{R}^{N} \text { and a.e. } t \in[0, T]
$$

(K2) $(\nabla K(t, x), x) \leq 2 K(t, x)$ for all $x \in \mathbb{R}^{N}$ and a.e. $t \in[0, T]$;

(K3) There exists $D_{1}>0$ such that

$$
K(t, x) \leq D_{1}|x|^{2} \quad \text { for all } x \in \mathbb{R}^{N} \text { and a.e. } t \in[0, T]
$$

(W1) $\lim _{|x| \rightarrow 0} \frac{W(t, x)}{|x|^{2}}<a$ uniformly for a.e. $t \in[0, T]$;

(W2) There exist constants $b>0$ and $d>0$ such that

$$
W(t, x) \leq b|x|^{2}+d \quad \text { for all } x \in \mathbb{R}^{N} \text { and a.e. } t \in[0, T]
$$

(W3) There exists a function $h \in L^{1}(0, T ; \mathbb{R})$ such that

$$
e^{Q(t)}[(\nabla W(t, x), x)-2 W(t, x)] \geq h(t) \quad \text { for all } x \in \mathbb{R}^{N} \text { and a.e. } t \in[0, T]
$$

and

$$
\lim _{|x| \rightarrow \infty} e^{Q(t)}[(\nabla W(t, x), x)-2 W(t, x)]=+\infty \quad \text { for a.e. } t \in[0, T]
$$

where $Q(t)=\int_{0}^{t} q(s) d s ;$

(W4) There exists $x_{0} \in \mathbb{R}^{N}$ such that

$$
\int_{0}^{T} e^{Q(t)}\left[\frac{1}{2}\left(A(t) x_{0}, x_{0}\right)+K\left(t, x_{0}\right)-W\left(t, x_{0}\right)-\frac{h(t)}{2}\right] d t<0
$$

(W5) There exists a constant $D_{2}>\frac{\|A\|}{2}+D_{1}$ such that

$$
W(t, x) \geq D_{2}|x|^{2} \quad \text { for all } x \in \mathbb{R}^{N} \text { and a.e. } t \in[0, T] ;
$$

(I1) There exist constants $l_{j}>0(j=1, \ldots, p)$ such that

$$
I_{j}(x) \leq l_{j} \quad(j=1, \ldots, p) \text { for all } x \in \mathbb{R}^{N}
$$

(I2) $\sum_{j=1}^{p} e^{Q\left(t_{j}\right)} I_{j}(0)=0$ and $\sum_{j=1}^{p} e^{Q\left(t_{j}\right)} I_{j}(x) \geq 0$ for all $x \in \mathbb{R}^{N}$;

(I3) There exists a constant $C$ such that

$$
\sum_{j=1}^{p} e^{Q\left(t_{j}\right)}\left[2 I_{j}(x)-\left(\nabla I_{j}(x), x\right)\right] \geq C \quad \text { for all } x \in \mathbb{R}^{N}
$$


This paper is organized as follows. In Section 2, we present the definition of a subharmonic classical solution, a subharmonic weak solution and the variational structure for system (1.1) and make some preliminaries. In Section 3, we present our main theorems and their proofs. In Section 4, an example is given to verify our main theorems.

\section{Preliminaries}

In this section, we present the variational structure of system (1.1), which is motivated by $[15-17,28]$ and $[29]$.

Let

$$
H_{k T}^{1}=\left\{u: \mathbb{R} \rightarrow \mathbb{R}^{N} \mid u \text { is absolutely continuous, } u(t+k T)=u(t) \text { and } \dot{u} \in L^{2}(0, k T)\right\} .
$$

Define

$$
\langle u, v\rangle=\int_{0}^{k T} e^{Q(t)}(u(t), v(t)) d t+\int_{0}^{k T} e^{Q(t)}(P(t) \dot{u}(t), \dot{v}(t)) d t
$$

and

$$
\|u\|=\left[\int_{0}^{k T} e^{Q(t)}|u(t)|^{2} d t+\int_{0}^{k T} e^{Q(t)}(P(t) \dot{u}(t), \dot{u}(t)) d t\right]^{1 / 2}
$$

for each $u, v \in H_{k T}^{1}$. Then $\left(H_{k T}^{1},\langle\cdot, \cdot\rangle\right)$ is a Hilbert space. It is well known that

$$
\|u\|_{H_{k T}^{1}}=\left[\int_{0}^{k T}|u(t)|^{2} d t+\int_{0}^{k T}|\dot{u}(t)|^{2} d t\right]^{1 / 2}
$$

is also a norm on $H_{k T}^{1}$. Obviously, if the condition (P) holds, $\|u\|_{H_{k T}^{1}}$ and $\|u\|$ are equivalent. Moreover, there exists $C_{0 k}>0$ such that

$$
\|u\|_{\infty}=\max _{t \in[0, k T]}|u(t)| \leq C_{0 k}\|u\|_{H_{k T}^{1}}
$$

(see Proposition 1.1 in [1]). Hence, there exist positive constants $C_{1 k}, C_{2 k}$ such that

$$
\|u\|_{\infty} \leq C_{1 k}\|u\|, \quad\|u\|_{H_{k T}^{1}} \geq C_{2 k}\|u\|
$$

For any $a, b \in \mathbb{R}$, define

$$
\begin{aligned}
H^{2}(a, b)= & \left\{u: \mathbb{R} \rightarrow \mathbb{R}^{N} \mid \text { both } u \text { and } \dot{u} \text { are absolutely continuous on }(a, b),\right. \\
& \text { and } \left.\ddot{u} \in L^{2}(a, b)\right\}, \\
H^{2}[a, b)= & \left\{u: \mathbb{R} \rightarrow \mathbb{R}^{N} \mid \text { both } u \text { and } \dot{u} \text { are absolutely continuous on }[a, b),\right. \\
& \text { and } \left.\ddot{u} \in L^{2}(a, b)\right\}, \\
H^{2}(a, b]= & \left\{u: \mathbb{R} \rightarrow \mathbb{R}^{N} \mid \text { both } u \text { and } \dot{u} \text { are absolutely continuous on }(a, b],\right. \\
& \text { and } \left.\ddot{u} \in L^{2}(a, b)\right\} .
\end{aligned}
$$

If $u \in H_{k T}^{1}$, then $\dot{u}\left(t^{+}\right)-\dot{u}\left(t^{-}\right)=0$ may not hold, which leads to impulsive effects. 
Definition 2.1 Assume that $u \in H_{k T}^{1} \cap H^{2}\left[0, t_{1}\right) \cap\left(\bigcap_{j=1}^{p-1} H^{2}\left(t_{j}, t_{j+1}\right)\right) \cap H^{2}\left(t_{p}, T\right] \cap H^{2}[T, k T]$ and the limits $\dot{u}\left(t_{j}^{+}\right)$and $\dot{u}\left(t_{j}^{-}\right)(j=1,2, \ldots, p)$ exist. If $u$ satisfies system (1.1), then we say that $u$ is a subharmonic classical solution of system (1.1).

Remark 2.1 In [32], impulsive effects may occur periodically in $t_{j}, j \in\{1, \ldots, p\}$. In order to obtain a sequence of distinct subharmonic weak solutions (see Theorem 3.2 below), different from [32], in Definition 2.1, we assume that the impulsive effects only occur in $t_{j}$, $j=1, \ldots, p$, which belong to $(0, T)$. In other words, $u$ is absolutely continuous on $\mathbb{R}$ and $\dot{u}$ is absolutely continuous on $\left[0, t_{1}\right) \cup\left(\bigcup_{j=1}^{p-1}\left(t_{j}, t_{j+1}\right)\right) \cup\left(t_{p}, T\right] \cup[T, k T]$. Moreover, note that $u(t)=u(t+k T)$. Then it is easy to see that $\dot{u}(0)=\dot{u}(k T)$.

Note that $Q(t)=\int_{0}^{t} q(s) d s$. Then, by $T$-periodicity of $q$, we have $Q(k T)=k \int_{0}^{T} q(t) d t=0$. Moreover, obviously, $Q(t)$ is continuous on $\mathbb{R}$. We transform system (1.1) into the following system:

$$
\left\{\begin{array}{l}
\frac{d\left(e^{Q(t)} P(t) \dot{u}(t)\right)}{d t}+e^{Q(t)} B \dot{u}(t)+e^{Q(t)}\left(\frac{1}{2} q(t) B-A(t)\right) u(t)+e^{Q(t)} \nabla F(t, u(t)) \\
=0, \quad \text { a.e. } t \in \mathbb{R}, \\
\Delta\left(P\left(t_{j}\right) \dot{u}\left(t_{j}\right)\right)=P\left(t_{j}\right) \dot{u}\left(t_{j}^{+}\right)-P\left(t_{j}\right) \dot{u}\left(t_{j}^{-}\right)=\nabla I_{j}\left(u\left(t_{j}\right)\right), \quad j=1, \ldots, p .
\end{array}\right.
$$

Then system (2.2) is equivalent to system (1.1) and its solutions are the solutions of system (1.1).

By the idea in [15], we take $v \in H_{k T}^{1}$ and multiply the two sides of the equality

$$
\frac{d\left(e^{Q(t)} P(t) \dot{u}(t)\right)}{d t}+e^{Q(t)} B \dot{u}(t)+e^{Q(t)}\left(\frac{1}{2} q(t) B-A(t)\right) u(t)+e^{Q(t)} \nabla F(t, u(t))=0
$$

by $v$ and integrate it from 0 to $k T$. Then we obtain

$$
\begin{aligned}
& \int_{0}^{k T}\left(\frac{d\left(e^{Q(t)} P(t) \dot{u}(t)\right)}{d t}+e^{Q(t)} B \dot{u}(t)+e^{Q(t)}\left(\frac{1}{2} q(t) B-A(t)\right) u(t)\right. \\
& \left.\quad+e^{Q(t)} \nabla F(t, u(t)), v(t)\right) d t=0 .
\end{aligned}
$$

Note that $P(t+T)=P(t), P(t)$ is continuous on $\mathbb{R}$ and $\dot{u}(0)=\dot{u}(k T)$. By integration by parts and the continuity of $v$, we obtain

$$
\begin{aligned}
\int_{0}^{k T} & \left(\frac{d\left(e^{Q(t)} P(t) \dot{u}(t)\right)}{d t}, v(t)\right) d t \\
= & \sum_{j=0}^{p} \int_{t_{j}}^{t_{j+1}}\left(\frac{d\left(e^{Q(t)} P(t) \dot{u}(t)\right)}{d t}, v(t)\right) d t+\int_{T}^{k T}\left(\frac{d\left(e^{Q(t)} P(t) \dot{u}(t)\right)}{d t}, v(t)\right) d t \\
= & \sum_{j=0}^{p}\left[\left(e^{Q\left(t_{j+1}\right)} P\left(t_{j+1}\right) \dot{u}\left(t_{j+1}^{-}\right), v\left(t_{j+1}\right)\right)-\left(e^{Q\left(t_{j}\right)} P\left(t_{j}\right) \dot{u}\left(t_{j}^{+}\right), v\left(t_{j}\right)\right)\right. \\
& \left.-\int_{t_{j}}^{t_{j+1}}\left(e^{Q(t)} P(t) \dot{u}(t), \dot{v}(t)\right) d t\right] \\
& +\left(e^{Q(k T)} P(k T) \dot{u}(k T), v(k T)\right)-\left(e^{Q(T)} P(T) \dot{u}(T), v(T)\right)
\end{aligned}
$$




$$
\begin{aligned}
& -\int_{T}^{k T}\left(e^{Q(t)} P(t) \dot{u}(t), \dot{v}(t)\right) d t \\
= & \left(e^{Q(T)} P(T) \dot{u}(T), v(T)\right)-\left(e^{Q(0)} P(0) \dot{u}(0), v(0)\right) \\
& -\sum_{j=1}^{p}\left[\left(e^{Q\left(t_{j}\right)} P\left(t_{j}\right) \dot{u}\left(t_{j}^{+}\right), v\left(t_{j}\right)\right)-\left(e^{Q\left(t_{j}\right)} P\left(t_{j}\right) \dot{u}\left(t_{j}^{-}\right), v\left(t_{j}\right)\right)\right] \\
& -\int_{0}^{T}\left(e^{Q(t)} P(t) \dot{u}(t), \dot{v}(t)\right) d t \\
& +\left(e^{Q(k T)} P(k T) \dot{u}(k T), v(k T)\right)-\left(e^{Q(T)} P(T) \dot{u}(T), v(T)\right) \\
& -\int_{T}^{k T}\left(e^{Q(t)} P(t) \dot{u}(t), \dot{v}(t)\right) d t \\
= & \left(e^{Q(k T)} P(k T) \dot{u}(k T), v(k T)\right)-\left(e^{Q(0)} P(0) \dot{u}(0), v(0)\right) \\
& -\sum_{j=1}^{p}\left[\left(e^{Q\left(t_{j}\right)} P\left(t_{j}\right) \dot{u}\left(t_{j}^{+}\right), v\left(t_{j}\right)\right)-\left(e^{Q\left(t_{j}\right)} P\left(t_{j}\right) \dot{u}\left(t_{j}^{-}\right), v\left(t_{j}\right)\right)\right] \\
& -\int_{0}^{k T}\left(e^{Q(t)} P(t) \dot{u}(t), \dot{v}(t)\right) d t \\
= & -\sum_{j=1}^{p} e^{Q\left(t_{j}\right)}\left(\Delta\left(P\left(t_{j}\right) \dot{u}\left(t_{j}\right)\right), v\left(t_{j}\right)\right)-\int_{0}^{k T}\left(e^{Q(t)} P(t) \dot{u}(t), \dot{v}(t)\right) d t .
\end{aligned}
$$

Definition 2.2 $u \in H_{k T}^{1}$ is called a subharmonic weak solution of system (1.1) if

$$
\begin{gathered}
\int_{0}^{k T}\left(e^{Q(t)} P(t) \dot{u}(t), \dot{v}(t)\right) d t+\sum_{j=1}^{p} e^{Q\left(t_{j}\right)}\left(\nabla I_{j}\left(u\left(t_{j}\right)\right), v\left(t_{j}\right)\right) \\
=\int_{0}^{k T} e^{Q(t)}\left[\frac{1}{2} q(t)(B u(t), v(t))+(B \dot{u}(t), v(t))\right. \\
\quad-(A(t) u(t), v(t))+(\nabla F(t, u(t)), v(t))] d t
\end{gathered}
$$

holds for any $v \in H_{k T}^{1}$.

Lemma 2.1 If $u \in H_{k T}^{1}$ is a subharmonic weak solution of system (1.1), then $u$ is a subharmonic classical solution of system (1.1).

Proof Motivated by [15], for $j \in\{0,1,2, \ldots, p\}$, choose $v \in H_{k T}^{1}$ with $v(t)=0$ for every $t \in$ $\left[0, t_{j}\right] \cup\left[t_{j+1}, k T\right]$. Then, by Definition 2.2 , we obtain

$$
\begin{aligned}
& \int_{t_{j}}^{t_{j+1}}\left(e^{Q(t)} P(t) \dot{u}(t), \dot{v}(t)\right) d t \\
& =\int_{t_{j}}^{t_{j+1}} e^{Q(t)}\left[\frac{1}{2} q(t)(B u(t), v(t))+(B \dot{u}(t), v(t))\right. \\
& \quad-(A(t) u(t), v(t))+(\nabla F(t, u(t)), v(t))] d t .
\end{aligned}
$$


Choose $v \in H_{k T}^{1}$ with $v(t)=0$ for every $t \in[0, T]$. Then we obtain

$$
\begin{aligned}
\int_{T}^{k T} & \left(e^{Q(t)} P(t) \dot{u}(t), \dot{v}(t)\right) d t \\
= & \int_{T}^{k T} e^{Q(t)}\left[\frac{1}{2} q(t)(B u(t), v(t))+(B \dot{u}(t), v(t))\right. \\
& \quad-(A(t) u(t), v(t))+(\nabla F(t, u(t)), v(t))] d t .
\end{aligned}
$$

Equations (2.5) and (2.6) imply that $u \in H_{k T}^{1} \cap H^{2}\left[0, t_{1}\right) \cap\left(\bigcap_{j=1}^{p-1} H^{2}\left(t_{j}, t_{j+1}\right)\right) \cap H^{2}\left(t_{p}, T\right] \cap$ $H^{2}[T, k T]$ and $u$ satisfies

$$
\begin{aligned}
& \frac{d\left(e^{Q(t)} P(t) \dot{u}(t)\right)}{d t}+e^{Q(t)} B \dot{u}(t)+e^{Q(t)}\left(\frac{1}{2} q(t) B-A(t)\right) u(t)+e^{Q(t)} \nabla F(t, u(t)) \\
& =0, \quad \text { a.e. } t \in[0, k T] .
\end{aligned}
$$

Multiplying the above equality by $v$ and integrating between 0 and $k T$, combining the argument of (2.4) and Definition 2.2, we obtain that

$$
\sum_{j=1}^{p} e^{Q\left(t_{j}\right)}\left(\Delta\left(P\left(t_{j}\right) \dot{u}\left(t_{j}\right)\right), v\left(t_{j}\right)\right)=\sum_{j=1}^{p} e^{Q\left(t_{j}\right)}\left(\nabla I_{j}\left(u\left(t_{j}\right)\right), v\left(t_{j}\right)\right) .
$$

Hence, $\Delta\left(P\left(t_{j}\right) \dot{u}\left(t_{j}\right)\right)=\nabla I_{j}\left(u\left(t_{j}\right)\right)$ for every $j=1,2, \ldots, p$. This completes the proof.

For every $k \in \mathbb{N}$, define $\varphi_{k}: H_{k T}^{1} \rightarrow \mathbb{R}$ by

$$
\begin{aligned}
\varphi_{k}(u)= & \int_{0}^{k T} e^{Q(t)}\left[\frac{1}{2}(P(t) \dot{u}(t), \dot{u}(t))+\frac{1}{2}(B u(t), \dot{u}(t))\right. \\
& \left.+\frac{1}{2}(A(t) u(t), u(t))-F(t, u(t))\right] d t+\sum_{j=1}^{p} e^{Q\left(t_{j}\right)} I_{j}\left(u\left(t_{j}\right)\right) .
\end{aligned}
$$

It follows from assumption (A) and Theorem 1.4 in [1] that the functional $\varphi_{k}$ is continuously differentiable and

$$
\begin{aligned}
\left\langle\varphi_{k}^{\prime}(u), v\right\rangle= & \int_{0}^{k T} e^{Q(t)}\left[(P(t) \dot{u}(t), \dot{v}(t))-\frac{1}{2} q(t)(B u(t), v(t))-(B \dot{u}(t), v(t))\right. \\
& +(A(t) u(t), v(t))-(\nabla F(t, u(t)), v(t))] d t+\sum_{j=1}^{p} e^{Q\left(t_{j}\right)}\left(\nabla I_{j}\left(u\left(t_{j}\right)\right), v\left(t_{j}\right)\right)
\end{aligned}
$$

for $u, v \in H_{k T}^{1}$. Obviously, if $u_{0} \in H_{k T}^{1}$ is a critical point of $\varphi_{k}$, i.e., $\varphi_{k}^{\prime}\left(u_{0}\right)=0$, then $u_{0}$ is a subharmonic weak solution of system (1.1).

We will use the following mountain pass theorem to prove our results.

Lemma 2.2 (see [30]) Let $E$ be a real Banach space, and let $\phi \in C^{1}(E, \mathbb{R})$ satisfy the (PS) condition. If $\phi$ satisfies the following conditions:

(i) $\phi(0)=0$;

(ii) There exist constants $\rho, \alpha>0$ such that $\left.\phi\right|_{\partial B_{\rho}(0)} \geq \alpha$; 
(iii) There exists $e \in E / \bar{B}_{\rho}(0)$ such that $\phi(e) \leq 0$, then $\phi$ possesses a critical value $c \geq \alpha$ given by

$$
c=\inf _{g \in \Gamma} \max _{s \in[0,1]} \phi(g(s))
$$

where $B_{\rho}(0)$ is an open ball in $E$ of radius $\rho$ centered at 0 , and

$$
\Gamma=\{g \in C([0,1], E): g(0)=0, g(1)=e\} .
$$

Remark 2.2 As shown in [31], a deformation lemma can be proved by replacing the usual (PS)-condition with the condition (C), and it turns out that Lemma 2.2 holds true under the condition (C). We say that $\phi$ satisfies the condition (C), i.e., for every sequence $\left\{u_{n}\right\} \subset$ $E,\left\{u_{n}\right\}$ has a convergent subsequence if $\phi\left(u_{n}\right)$ is bounded and $\left(1+\left\|u_{n}\right\|\right)\left\|\phi^{\prime}\left(u_{n}\right)\right\| \rightarrow 0$ as $n \rightarrow \infty$.

\section{Main results}

Theorem 3.1 Assume that (P), (K1), (K2), (W1)-(W4) and (I1)-(I3) hold. Then, for every $k \in \mathbb{N}$, system (1.1) has at least one $k T$-periodic weak solution in $H_{k T}^{1}$.

Proof We use Lemma 2.2 to prove the theorem. Let $E=H_{k T}^{1}$.

Step 1. We prove that $\varphi_{k}$ satisfies assumption (ii) of Lemma 2.2. It follows from (W1) and (W2) that there exist $0<\varepsilon<\frac{m}{2}-\frac{1}{4}, \theta>2$ and $C_{1}>0$ such that

$$
W(t, x) \leq(a-\varepsilon)|x|^{2}+C_{1}|x|^{\theta} .
$$

Choose $0<\delta<1$ such that $\varepsilon\left(\frac{\delta}{C_{1 k}}\right)^{2}-C_{1} C_{1 k}^{\theta}\left(\frac{\delta}{C_{1 k}}\right)^{\theta} \int_{0}^{k T} e^{Q(t)} d t>0$. Then it follows from (K1), (I2), (3.1) and (2.1) that for all $u \in H_{k T}^{1}$ with $\|u\|=\delta / C_{1 k}:=\rho_{k}$,

$$
\begin{aligned}
\varphi_{k}(u)= & \int_{0}^{k T} e^{Q(t)}\left[\frac{1}{2}(P(t) \dot{u}(t), \dot{u}(t))+\frac{1}{2}(B u(t), \dot{u}(t))+\frac{1}{2}(A(t) u(t), u(t))\right] d t \\
& +\int_{0}^{k T} e^{Q(t)}[K(t, u(t))-W(t, u(t))] d t+\sum_{j=1}^{p} e^{Q\left(t_{j}\right)} I_{j}\left(u\left(t_{j}\right)\right) \\
\geq & \int_{0}^{k T} e^{Q(t)}\left[\frac{m}{2}|\dot{u}(t)|^{2}-\frac{1}{4}\left(\|B\|^{2}|u(t)|^{2}+|\dot{u}(t)|^{2}\right)-\frac{\|A\||u(t)|^{2}}{2}\right] d t \\
& +\left(\frac{\|B\|^{2}+2\|A\|}{4}+a\right) \int_{0}^{k T} e^{Q(t)}|u(t)|^{\gamma} d t-(a-\varepsilon) \int_{0}^{k T} e^{Q(t)}|u(t)|^{2} d t \\
& -C_{1} \int_{0}^{k T} e^{Q(t)}|u(t)|^{\theta} d t \\
\geq & \left(\frac{m}{2}-\frac{1}{4}\right) \int_{0}^{k T} e^{Q(t)}|\dot{u}(t)|^{2} d t+a \int_{0}^{k T} e^{Q(t)}|u(t)|^{\gamma} d t \\
& -(a-\varepsilon) \int_{0}^{k T} e^{Q(t)}|u(t)|^{2} d t-C_{1} \int_{0}^{k T} e^{Q(t)}|u(t)|^{\theta} d t \\
\geq & \left(\frac{m}{2}-\frac{1}{4}\right) \int_{0}^{k T} e^{Q(t)}|\dot{u}(t)|^{2} d t+\varepsilon \int_{0}^{k T} e^{Q(t)}|u(t)|^{2} d t
\end{aligned}
$$




$$
\begin{aligned}
& -C_{1}\|u\|_{\infty}^{\theta} \int_{0}^{k T} e^{Q(t)} d t \\
\geq & \varepsilon\|u\|^{2}-C_{1} C_{1 k}^{\theta}\|u\|^{\theta} \int_{0}^{k T} e^{Q(t)} d t \\
= & \varepsilon\left(\frac{\delta}{C_{1 k}}\right)^{2}-C_{1} C_{1 k}^{\theta}\left(\frac{\delta}{C_{1 k}}\right)^{\theta} \int_{0}^{k T} e^{Q(t)} d t \\
= & : \alpha_{k}>0 .
\end{aligned}
$$

Step 2. We prove that $\varphi_{k}$ satisfies assumption (iii) of Lemma 2.2. Set $\varphi(s)=s^{-2} W\left(t, s x_{0}\right)$ for $s>0$. By the argument in [10], we know that (W3) implies that

$$
W\left(t, s x_{0}\right) \geq s^{2} W\left(t, x_{0}\right)+\frac{h(t)}{2}\left(s^{2}-1\right) \quad \text { for a.e. } t \in[0, T], s>1
$$

and (K2) implies that

$$
K\left(t, s x_{0}\right) \leq s^{2} K\left(t, x_{0}\right) \quad \text { for a.e. } t \in[0, T], s>1
$$

It follows from (3.2), (3.3), (W3) and (I1) that for sufficiently large $s$,

$$
\begin{aligned}
\varphi_{k}\left(s x_{0}\right)= & \frac{s^{2}}{2} \int_{0}^{k T} e^{Q(t)}\left(A(t) x_{0}, x_{0}\right) d t+\int_{0}^{k T} e^{Q(t)}\left[K\left(t, s x_{0}\right)-W\left(t, s x_{0}\right)\right] d t \\
& +\sum_{j=1}^{p} e^{Q\left(t_{j}\right)} I_{j}\left(s x_{0}\right) \\
= & \frac{s^{2}}{2} k \int_{0}^{T} e^{Q(t)}\left(A(t) x_{0}, x_{0}\right) d t+k \int_{0}^{T} e^{Q(t)}\left[K\left(t, s x_{0}\right)-W\left(t, s x_{0}\right)\right] d t \\
& +\sum_{j=1}^{p} e^{Q\left(t_{j}\right)} I_{j}\left(s x_{0}\right) \\
\leq & s^{2} k \int_{0}^{T} e^{Q(t)}\left[\frac{1}{2}\left(A(t) x_{0}, x_{0}\right)+K\left(t, x_{0}\right)-W\left(t, x_{0}\right)-\frac{h(t)}{2}\right] d t \\
& +\frac{1}{2} \int_{0}^{k T} e^{Q(t)} h(t) d t+\sum_{j=1}^{p} e^{Q\left(t_{j}\right)} l_{j} .
\end{aligned}
$$

By (W4), we can choose sufficiently large $s_{0 k}$ such that $\left\|s_{0 k} x_{0}\right\|>\rho_{k}$ and $\varphi_{k}\left(s_{0 k} x_{0}\right) \leq 0$. Let $e_{k}=s_{0 k} x_{0}$. Then $\varphi_{k}$ satisfies assumption (iii) of Lemma 2.2.

Step 3. We prove that $\varphi$ satisfies the (C)-condition on $H_{k T}^{1}$. The proof is motivated by [10]. For every $\left\{u_{n}\right\} \subset H_{k T}^{1}$, assume that there exists a constant $C_{3 k}>0$ such that

$$
\left|\varphi_{k}\left(u_{n}\right)\right| \leq C_{3 k}, \quad\left(1+\left\|u_{n}\right\|\right)\left\|\varphi_{k}^{\prime}\left(u_{n}\right)\right\| \leq C_{3 k} \quad \text { for all } n \in \mathbb{N}
$$

Then it follows from antisymmetry of $B,(\mathrm{~K} 2)$ and (I3) that

$$
\begin{aligned}
3 C_{3 k} & \geq 2 \varphi_{k}\left(u_{n}\right)-\left(\varphi_{k}^{\prime}\left(u_{n}\right), u_{n}\right) \\
& =\frac{1}{2} \int_{0}^{k T} e^{Q(t)} q(t)\left(B u_{n}(t), u_{n}(t)\right) d t
\end{aligned}
$$




$$
\begin{aligned}
& \quad+\int_{0}^{k T} e^{Q(t)}\left[\left(\nabla F\left(t, u_{n}(t)\right), u_{n}(t)\right)-2 F\left(t, u_{n}(t)\right)\right] d t \\
& +\sum_{j=1}^{p} e^{Q\left(t_{j}\right)}\left[2 I_{j}\left(u_{n}\left(t_{j}\right)\right)-\left(\nabla I_{j}\left(u_{n}\left(t_{j}\right)\right), u_{n}\left(t_{j}\right)\right)\right] \\
& =\int_{0}^{k T} e^{Q(t)}\left[2 K\left(t, u_{n}(t)\right)-\left(\nabla K\left(t, u_{n}(t)\right), u_{n}(t)\right)\right] d t \\
& \quad+\int_{0}^{k T} e^{Q(t)}\left[\left(\nabla W\left(t, u_{n}(t)\right), u_{n}(t)\right)-2 W\left(t, u_{n}(t)\right)\right] d t \\
& \quad+\sum_{j=1}^{p} e^{Q\left(t_{j}\right)}\left[2 I_{j}\left(u_{n}\left(t_{j}\right)\right)-\left(\nabla I_{j}\left(u_{n}\left(t_{j}\right)\right), u_{n}\left(t_{j}\right)\right)\right] \\
& \geq \int_{0}^{k T} e^{Q(t)}\left[\left(\nabla W\left(t, u_{n}(t)\right), u_{n}(t)\right)-2 W\left(t, u_{n}(t)\right)\right] d t+C .
\end{aligned}
$$

Next we prove that $\left\{u_{n}\right\}$ is bounded. Assume that $\left\|u_{n}\right\| \rightarrow \infty$ as $n \rightarrow \infty$. Let $z_{n}=\frac{u_{n}}{\left\|u_{n}\right\|}$. Then $\left\|z_{n}\right\|=1$, and so there exists a subsequence, still denoted by $\left\{z_{n}\right\}$, such that $z_{n} \rightarrow z$ on $H_{k T}^{1}$. Then, by Proposition 1.2 in [1], we get $\left\|z_{n}-z\right\|_{\infty} \rightarrow 0$. Hence, we have $\int_{0}^{k T} \mid z_{n}(t)-$ $\left.z(t)\right|^{2} d t \rightarrow 0$ and $z_{n}(t) \rightarrow z(t)$ for a.e. $t \in[0, k T]$. Thus, by conditions (P), (W2) and (I2), we have

$$
\begin{aligned}
\varphi_{k}\left(u_{n}\right)= & \int_{0}^{k T} e^{Q(t)}\left[\frac{1}{2}\left(P(t) \dot{u}_{n}(t), \dot{u}_{n}(t)\right)+\frac{1}{2}\left(B u_{n}(t), \dot{u}_{n}(t)\right)\right. \\
& \left.+\frac{1}{2}\left(A(t) u_{n}(t), u_{n}(t)\right)+K\left(t, u_{n}(t)\right)-W\left(t, u_{n}(t)\right)\right] d t \\
& +\sum_{j=1}^{p} e^{Q\left(t_{j}\right)} I_{j}\left(u_{n}\left(t_{j}\right)\right) \\
\geq & \int_{0}^{k T} e^{Q(t)}\left[\frac{1}{2}\left(P(t) \dot{u}_{n}(t), \dot{u}_{n}(t)\right)-\frac{\|B\|^{2}\left|u_{n}(t)\right|^{2}+\left|\dot{u}_{n}(t)\right|^{2}}{4}-\frac{\|A\|\left|u_{n}(t)\right|^{2}}{2}\right] d t \\
& -b \int_{0}^{k T} e^{Q(t)}\left|u_{n}(t)\right|^{2} d t-d \int_{0}^{k T} e^{Q(t)} d t \\
\geq & \left(\frac{1}{2}-\frac{1}{4 m}\right) \int_{0}^{k T} e^{Q(t)}\left[\left(P(t) \dot{u}_{n}(t), \dot{u}_{n}(t)\right)\right] d t \\
& -\frac{\|B\|^{2}+2\|A\|+4 b}{4} \int_{0}^{k T} e^{Q(t)}\left|u_{n}(t)\right|^{2} d t-d \int_{0}^{k T} e^{Q(t)} d t \\
= & \left(\frac{1}{2}-\frac{1}{4 m}\right)\left\|u_{n}\right\|^{2}-\left(\frac{1}{2}-\frac{1}{4 m}\right) \int_{0}^{k T} e^{Q(t)}\left|u_{n}(t)\right|^{2} d t \\
& -\frac{\|B\|^{2}+2\|A\|+4 b}{4} \int_{0}^{k T} e^{Q(t)}\left|u_{n}(t)\right|^{2} d t-d \int_{0}^{k T} e^{Q(t)} d t \\
= & \left(\frac{1}{2}-\frac{1}{4 m}\right)\left\|u_{n}\right\|^{2}-\left[\frac{\|B\|^{2}+2\|A\|+4 b}{4}+\left(\frac{1}{2}-\frac{1}{4 m}\right)\right] \int_{0}^{k T} e^{Q(t)}\left|u_{n}(t)\right|^{2} d t \\
& -d \int_{0}^{k T} e^{Q(t)} d t .
\end{aligned}
$$


Hence, we have

$$
\begin{aligned}
\frac{\varphi_{k}\left(u_{n}\right)}{\left\|u_{n}\right\|^{2}} \geq & \left(\frac{1}{2}-\frac{1}{4 m}\right)-\left[\frac{\|B\|^{2}+2\|A\|+4 b}{4}+\left(\frac{1}{2}-\frac{1}{4 m}\right)\right] \int_{0}^{k T} e^{Q(t)} \frac{\left|u_{n}(t)\right|^{2}}{\left\|u_{n}\right\|^{2}} d t \\
& -\frac{d \int_{0}^{k T} e^{Q(t)} d t}{\left\|u_{n}\right\|^{2}} .
\end{aligned}
$$

Let $n \rightarrow \infty$. Then, by (3.4), we get

$$
\frac{1}{2}-\frac{1}{4 m} \leq\left[\frac{\|B\|^{2}+2\|A\|+4 b}{4}+\left(\frac{1}{2}-\frac{1}{4 m}\right)\right] \int_{0}^{k T} e^{Q(t)}\left|z_{n}(t)\right|^{2} d t .
$$

Then it follows from $m>\frac{1}{2}$ and (3.6) that $\int_{0}^{k T} e^{Q(t)}|z(t)|^{2} d t>0$ and so $z \neq 0$. Let $S=\{t \in$ $\left.[0, k T]: \lim _{|x| \rightarrow \infty} e^{Q(t)}[2 W(t, x)-(\nabla W(t, x), x)]=+\infty\right\}$ and $S_{1}=\{t \in S: z(t) \neq 0\}$. Then meas $S_{1}>0$ and

$$
\lim _{n \rightarrow \infty}\left|u_{n}(t)\right|=+\infty \quad \text { for every } t \in S_{1}
$$

Let $f_{n}(t)=e^{Q(t)}\left[\left(\nabla W\left(t, u_{n}(t)\right), u_{n}(t)\right)-2 W\left(t, u_{n}(t)\right)\right]$. Then (3.7) and $T$-periodicity of $W(t, x)$ in $t$ imply that

$$
\lim _{n \rightarrow \infty} f_{n}(t)=+\infty \quad \text { for every } t \in S_{1}
$$

It follows from (3.8) and Lemma 1 in [6] that there exists a subset $S_{2}$ of $S_{1}$ with meas $S_{2}>0$ such that

$$
\lim _{n \rightarrow \infty} f_{n}(t)=+\infty \quad \text { uniformly for } t \in S_{2} \text {. }
$$

By (W3), we have

$$
\begin{aligned}
& \int_{0}^{k T} e^{Q(t)}\left[\left(\nabla W\left(t, u_{n}(t)\right), u_{n}(t)\right)-2 W\left(t, u_{n}(t)\right)\right] d t \\
& =\int_{S_{2}} e^{Q(t)}\left[\left(\nabla W\left(t, u_{n}(t)\right), u_{n}(t)\right)-2 W\left(t, u_{n}(t)\right)\right] d t \\
& \quad+\int_{[0, k T] / S_{2}} e^{Q(t)}\left[\left(\nabla W\left(t, u_{n}(t)\right), u_{n}(t)\right)-2 W\left(t, u_{n}(t)\right)\right] d t \\
& \geq \int_{S_{2}} e^{Q(t)}\left[\left(\nabla W\left(t, u_{n}(t)\right), u_{n}(t)\right)-2 W\left(t, u_{n}(t)\right)\right] d t+\int_{[0, k T] / S_{2}} h(t) d t .
\end{aligned}
$$

Let $n \rightarrow \infty$. Then by Fatou's lemma and (3.9), we have

$$
\int_{0}^{k T} e^{Q(t)}\left[\left(\nabla W\left(t, u_{n}(t)\right), u_{n}(t)\right)-2 W\left(t, u_{n}(t)\right)\right] d t \rightarrow+\infty
$$

which contradicts (3.5). Hence $\left\{u_{n}\right\}$ is bounded. Going if necessary to a subsequence, assume that $u_{n} \rightarrow u$ in $H_{k T}^{1}$. Then, by Proposition 1.2 in [1], we have $\left\|u_{n}-u\right\|_{\infty} \rightarrow 0$ and so $\int_{0}^{k T} e^{Q(t)}\left|u_{n}-u\right|^{2} d t \rightarrow 0$ as $n \rightarrow \infty$. Similar to the argument of Theorem 3.1 in [28], it is 
easy to obtain that $\int_{0}^{k T} e^{Q(t)}\left(P(t)\left(\dot{u}_{n}-\dot{u}\right), \dot{u}_{n}-\dot{u}\right) d t \rightarrow 0$. Hence, $\left\|u_{n}-u\right\| \rightarrow 0$ as $n \rightarrow \infty$. Hence, $\varphi_{k}$ satisfies the $(\mathrm{C})$-condition.

Finally, (K1), (W1) and (I2) imply that $\varphi_{k}(0)=0$. Hence, combining Step 1-Step 3 with Lemma 2.2 and Remark 2.2, we obtain that $\varphi_{k}$ has at least a critical point $u_{k}$ in $H_{k T}^{1}$ and $\varphi_{k}\left(u_{k}\right)=c_{k} \geq \alpha_{k}>0$. Then system (1.1) has at least one $k T$-periodic solution $u_{k}$ in $H_{k T}^{1}$. This completes the proof.

Remark 3.1 It is easy to see that Theorem 3.1 generalizes Theorem A. To be precise, when $k=1, P(t) \equiv I_{N}, q(t) \equiv 0, B=0, A(t) \equiv 0$ and $I_{j}(x) \equiv 0$, Theorem 3.1 reduces to Theorem A.

Theorem 3.2 Assume (P), (K1)-(K3), (W1)-(W5) and (I1)-(I3) hold. Then system (1.1) has a sequence of distinct subharmonic weak solutions with period $k_{j} T$ satisfying $k_{j} \in \mathbb{N}$ and $k_{j} \rightarrow \infty$ as $j \rightarrow \infty$.

Proof By Theorem 3.1, we know that for every $k \in \mathbb{N}$, system (1.1) has at least one $k T$ periodic solution $u_{k}$ in $H_{k T}^{1}$ and $\varphi_{k}\left(u_{k}\right)=c_{k} \geq \alpha_{k}>0$. By Lemma 2.2, we have

$$
\varphi_{k}\left(u_{k}\right)=c_{k}=\inf _{g \in \Gamma \in[0,1]} \max _{s \in[}(g(s))
$$

where

$$
\Gamma=\left\{g \in C([0,1], E): g(0)=0, g(1)=e_{k}\right\} .
$$

Let $g(s)=s e_{k}=s s_{0 k} x_{0}, s \in[0,1]$. Obviously, $g \in \Gamma$. Hence, by (K3), (W5) and (I1), we have

$$
\begin{aligned}
\varphi_{k}\left(u_{k}\right) \leq & \max _{s \in[0,1]} \varphi_{k}\left(s s_{0} x_{0}\right) \\
= & \max _{s \in[0,1]}\left\{\int_{0}^{k T} e^{Q(t)}\left[\frac{1}{2}\left(A(t) s s_{0} x_{0}, s s_{0} x_{0}\right)+K\left(t, s s_{0} x_{0}\right)-W\left(t, s s_{0} x_{0}\right)\right] d t\right. \\
& \left.+\sum_{j=1}^{p} e^{Q\left(t_{j}\right)} I_{j}\left(s s_{0} x_{0}\right)\right\} \\
= & \max _{s \in[0,1]}\left\{k \int_{0}^{T} e^{Q(t)}\left[\frac{1}{2}\left(A(t) s s_{0} x_{0}, s s_{0} x_{0}\right)+K\left(t, s s_{0} x_{0}\right)-W\left(t, s s_{0} x_{0}\right)\right] d t\right. \\
& \left.+\sum_{j=1}^{p} e^{Q\left(t_{j}\right)} I_{j}\left(s s_{0} x_{0}\right)\right\} \\
\leq & \max _{s \in[0,1]}\left\{k \int_{0}^{T} e^{Q(t)}\left[\frac{\|A\|}{2}\left|s s_{0} x_{0}\right|^{2}+D_{1}\left|s s_{0} x_{0}\right|^{2}-D_{2}\left|s s_{0} x_{0}\right|^{2}\right] d t+\sum_{j=1}^{p} e^{Q\left(t_{j}\right)} l_{j}\right\} \\
= & \max _{s \in[0,1]}\left\{\left[\frac{\|A\|}{2}+D_{1}-D_{2}\right] s^{2} s_{0}^{2}\left|x_{0}\right|^{2} k \int_{0}^{T} e^{Q(t)} d t+\sum_{j=1}^{p} e^{Q\left(t_{j}\right)} l_{j}\right\} \\
\leq & \sum_{j=1}^{p} e^{Q\left(t_{j}\right)} l_{j}:=M .
\end{aligned}
$$

Hence, $\varphi_{k}\left(u_{k}\right)$ is uniformly bounded for all $k \in \mathbb{N}$. 
Obviously, we can find $k_{1} \in \mathbb{N} /\{1\}$ such that $k_{1}>\frac{M}{\alpha_{1}}$, then we claim that $u_{k}$ is distinct to $u_{1}$ for all $k \geq k_{1}$. In fact, if $u_{k}=u_{1}$ for some $k \geq k_{1}$, it is easy to check that

$$
\varphi_{k}\left(u_{k}\right)=k \varphi_{1}\left(u_{1}\right) \geq k \alpha_{1} .
$$

Then, by (3.10), we have $k_{1} \leq k \leq \frac{M}{\alpha_{1}}$, a contradiction. We can also find $k_{2}>\max \left\{k_{1}, \frac{k_{1} M}{\alpha_{k_{1}}}\right\}$ such that $u_{k_{1} k} \neq u_{k_{1}}$ for all $k \geq \frac{k_{2}}{k_{1}}$. Otherwise, if $u_{k_{1} k}=u_{k_{1}}$ for some $k \geq k_{1}$, we have $\varphi_{k_{1} k}\left(u_{k_{1} k}\right)=k \varphi_{k_{1}}\left(u_{k_{1}}\right) \geq k \alpha_{k_{1}}$. Then by (3.10), we have $\frac{k_{2}}{k_{1}} \leq k \leq \frac{M}{\alpha_{k_{1}}}$, a contradiction. In the same way, we can obtain that system (1.1) has a sequence of distinct periodic solutions with period $k_{j} T$ satisfying $k_{j} \in \mathbb{N}$ and $k_{j} \rightarrow \infty$ as $j \rightarrow \infty$. This completes the proof.

\section{Example}

The following example is inspired partially by Example 3.1 in [10]. Let $T=2 \pi, N=3$. Consider the following impulsive Lagrangian system with damped term:

$$
\left\{\begin{array}{l}
\frac{d(P(t) \dot{u}(t))}{d t}+(q(t) P(t)+B) \dot{u}(t)+\left(\frac{1}{2} q(t) B-A(t)\right) u(t)+\nabla F(t, u(t)) \\
\quad=0, \quad \text { a.e. } t \in \mathbb{R}, \\
\Delta\left(P\left(\frac{\pi}{2}\right) \dot{u}\left(\frac{\pi}{2}\right)\right)=P\left(\frac{\pi}{2}\right) \dot{u}\left(\frac{\pi}{2}^{+}\right)-P\left(\frac{\pi}{2}\right) \dot{u}\left(\frac{\pi}{2}^{-}\right)=\nabla I_{1}\left(u\left(\frac{\pi}{2}\right)\right), \\
\Delta(P(\pi) \dot{u}(\pi))=P(\pi) \dot{u}\left(\pi^{+}\right)-P(\pi) \dot{u}\left(\pi^{-}\right)=\nabla I_{2}(u(\pi)),
\end{array}\right.
$$

where

$$
\begin{aligned}
& P(t)=\left(\begin{array}{ccc}
\sin t+2 & 0 & 0 \\
0 & \sin t+2 & 0 \\
0 & 0 & \cos t+2
\end{array}\right), \quad A(t)=\left(\begin{array}{ccc}
\cos t & 0 & 0 \\
0 & \cos t & 0 \\
0 & 0 & \cos t
\end{array}\right), \\
& B=\left(\begin{array}{ccc}
0 & 1 & 0 \\
-1 & 0 & 1 \\
0 & -1 & 0
\end{array}\right), \quad q(t)=\sin t, \quad K(t, x)=2\left(\left|\sin \frac{t}{2}\right|+6.5\right)|x|^{2}, \\
& W(t, x)=5\left(\left|\cos \frac{t}{2}\right|+1\right)|x|^{2}\left(2-\frac{1}{\ln \left(e+|x|^{2}\right)}\right), \\
& I_{1}(x)=\frac{|x|^{2}}{1+|x|^{2}}, \quad I_{2}(x)=\frac{\ln \left(1+|x|^{2}\right)}{|x|^{2}} .
\end{aligned}
$$

Obviously, the condition (P) holds and $\|A\|=1,\|B\|=\sqrt{2}$ and (K1), (K2), (W1) and (W2) hold with $a=11$ and $\gamma=2$.

$$
e^{Q(t)}[(\nabla W(t, x), x)-2 W(t, x)]=\frac{10\left(\left|\cos \frac{t}{2}\right|+1\right) e^{1-\cos t}|x|^{4}}{\left(e+|x|^{2}\right)\left[\ln \left(e+|x|^{2}\right)\right]^{2}} .
$$

Then (W3) holds with $h(t) \equiv 0$. Moreover,

$$
\begin{gathered}
\int_{0}^{2 \pi} e^{Q(t)}\left[\frac{1}{2}(A(t) x, x)+K(t, x)-W(t, x)-\frac{h(t)}{2}\right] d t \\
=\int_{0}^{2 \pi} e^{1-\cos t}\left[\frac{1}{2} \cos t|x|^{2}+2\left(\left|\sin \frac{t}{2}\right|+6.5\right)|x|^{2}\right.
\end{gathered}
$$




$$
\begin{aligned}
& \left.-5\left(\left|\cos \frac{t}{2}\right|+1\right)|x|^{2}\left(2-\frac{1}{\ln \left(e+|x|^{2}\right)}\right)\right] d t \\
\leq & e^{2} \int_{0}^{2 \pi}\left[\frac{1}{2} \cos t|x|^{2}+2\left(\left|\sin \frac{t}{2}\right|+6.5\right)|x|^{2}\right. \\
& \left.-5\left(\left|\cos \frac{t}{2}\right|+1\right)|x|^{2}\left(2-\frac{1}{\ln \left(e+|x|^{2}\right)}\right)\right] d t \\
= & e^{2}\left[(8+26 \pi)|x|^{2}-(20+10 \pi)|x|^{2}\left(2-\frac{1}{\ln \left(e+|x|^{2}\right)}\right)\right] .
\end{aligned}
$$

Hence, it is easy to see that there exists $x_{0} \in \mathbb{R}^{N}$ such that (W4) holds by the above inequality. Obviously, (I1) and (I2) hold. Note that

$$
\begin{aligned}
& \sum_{j=1}^{2} e^{Q\left(t_{j}\right)}\left[2 I_{j}(x)-\left(\nabla I_{j}(x), x\right)\right] \\
& \quad=e^{Q\left(\frac{\pi}{2}\right)}\left[\frac{2|x|^{2}}{1+|x|^{2}}-\frac{2|x|^{2}}{\left(1+|x|^{2}\right)^{2}}\right]+e^{Q(\pi)}\left[\frac{2 \ln \left(1+|x|^{2}\right)}{|x|^{2}}-\frac{\frac{2|x|^{2}}{1+|x|^{2}}-2 \ln \left(1+|x|^{2}\right)}{|x|^{2}}\right] \\
& \quad=\frac{2|x|^{2}}{1+|x|^{2}} e^{Q\left(\frac{\pi}{2}\right)}\left[1-\frac{1}{1+|x|^{2}}\right]+e^{Q(\pi)}\left[\frac{4 \ln \left(1+|x|^{2}\right)}{|x|^{2}}-\frac{2}{1+|x|^{2}}\right] \\
& \quad \geq-e^{Q(\pi)} \frac{2}{1+|x|^{2}} \\
& \quad \geq-2 e^{Q(\pi)} .
\end{aligned}
$$

So (I3) holds. Hence, by Theorem 3.1, we obtain that system (4.1) has at least one $k T$ periodic solution for every $k \in \mathbb{N}$.

Moreover, it is easy to see that (K3) holds with $D_{1}=4$. Since

$$
\begin{aligned}
W(t, x) & =5\left(\left|\cos \frac{t}{2}\right|+1\right)|x|^{2}\left(2-\frac{1}{\ln \left(e+|x|^{2}\right)}\right) \\
& \geq 5|x|^{2}\left(2-\frac{1}{\ln \left(e+|x|^{2}\right)}\right) \\
& \geq 5|x|^{2} .
\end{aligned}
$$

Choose $D_{2}=5$. Then (W5) holds. Hence, by Theorem 3.2, we obtain that system (4.1) has a sequence of distinct subharmonic weak solutions with period $k_{j} T$ satisfying $k_{j} \in \mathbb{N}$ and $k_{j} \rightarrow \infty$ as $j \rightarrow \infty$.

\section{Competing interests}

The author declares that he has no competing interests.

Author's contributions

The author read and approved the final manuscript.

\section{Acknowledgements}

This work is supported by Tianyuan Fund for Mathematics of the National Natural Science Foundation of China (No. 11226135) and the Fund for Fostering Talents in Kunming University of Science and Technology (No. KKSY201207032). 


\section{References}

1. Mawhin, J, Willem, M: Critical Point Theory and Hamiltonian Systems. Springer, New York (1989)

2. Long, YM: Nonlinear oscillations for classical Hamiltonian systems with bi-even subquadratic potentials. Nonlinear Anal. TMA 24, 1665-1671 (1995)

3. Ding, YH: Existence and multiplicity results for homoclinic solutions to a class of Hamiltonian systems. Nonlinear Anal. 25(11), 1095-1113 (1995)

4. Schechter, M: Periodic non-autonomous second-order dynamical systems. J. Differ. Equ. 223, 290-302 (2006)

5. Tang, CL: Periodic solutions of nonautonomous second order systems with $\gamma$-quasisubadditive potential. J. Math Anal. Appl. 189, 671-675 (1995)

6. Tang, CL, Wu, XP: Periodic solutions for second order systems with not uniformly coercive potential. J. Math. Anal. Appl. 259, 386-397 (2001)

7. Jiang, Q, Tang, CL: Periodic ad subharmonic solutions of a class of subquadratic second-order Hamiltonian systems. J. Math. Anal. Appl. 328, 380-389 (2007)

8. Wu, X: Saddle point characterization and multiplicity of periodic solutions of non-autonomous second-order systems. Nonlinear Anal. 58, 899-907 (2004)

9. Zhao, F, Wu, X: Existence and multiplicity of nonzero periodic solution with saddle point character for some nonautonomous second order systems. J. Math. Anal. Appl. 308, 588-595 (2005)

10. Tang, XH, Jiang, J: Existence and multiplicity of periodic solutions for a class of second-order Hamiltonian systems. Comput. Math. Appl. 59, 3646-3655 (2010)

11. Zhang, X, Zhou, Y: Periodic solutions of non-autonomous second order Hamiltonian systems. J. Math. Anal. Appl. 345, 929-933 (2008)

12. Zhang, $X$, Tang, $X$ : Subharmonic solutions for a class of non-quadratic second order Hamiltonian systems. Nonlinear Anal., Real World Appl. 13, 113-130 (2012)

13. Zhang, $X$, Tang, $X$ : Existence of subharmonic solutions for non-quadratic second order Hamiltonian systems. Bound. Value Probl. (2013). doi:10.1186/1687-2770-2013-139

14. Zhang, Q, Liu, C: Infinitely many homoclinic solutions for second order Hamiltonian systems. Nonlinear Anal. 72 894-903 (2010)

15. Nieto, JJ, O'Regan, D: Variational approach to impulsive differential equations. Nonlinear Anal., Real World Appl. 10, 680-690 (2009)

16. Nieto, JJ: Variational formulation of a damped Dirichlet impulsive problem. Appl. Math. Lett. 23, 940-942 (2010)

17. Xiao, J, Nieto, JJ: Variational approach to some damped Dirichlet nonlinear impulsive differential equations. J. Franklin Inst. 348, 369-377 (2011)

18. Xiao, J, Nieto, JJ, Luo, Z: Multiplicity of solutions for nonlinear second order impulsive differential equations with linear derivative dependence via variational methods. Commun. Nonlinear Sci. Numer. Simul. 17, 426-432 (2012)

19. Tian, Y, Ge, WG: Applications of variational methods to boundary value problem for impulsive differential equations. Proc. Edinb. Math. Soc. 51, 509-527 (2008)

20. Tian, Y, Ge, WG: Variational methods to Sturm-Liouville boundary value problem for impulsive differential equations. Nonlinear Anal. 72, 277-287 (2010)

21. Zhou, J, Li, Y: Existence of solutions for a class of second-order Hamiltonian systems with impulsive effects. Nonlinear Anal. 72, 1594-1603 (2010)

22. Sun, J, Chen, H, Nieto, JJ, Otero-Novoa, M: Multiplicity of solutions for perturbed second-order Hamiltonian systems with impulsive effects. Nonlinear Anal. 72, 4575-4586 (2010)

23. Sun, J, Chen, H, Nieto, JJ: Infinitely many solutions for second-order Hamiltonian system with impulsive effects. Math. Comput. Model. 54, 544-555 (2011)

24. Wu, X, Chen, S, Teng, K: On variational methods for a class of damped vibration problems. Nonlinear Anal. 68, 1432-1441 (2008)

25. Duan, $\mathrm{S}, \mathrm{Wu}, \mathrm{X}$ : The local linking theorem with an application to a class of second-order systems. Nonlinear Anal. 72 , 2488-2498 (2010)

26. Han, ZQ, Wang, SQ: Multiple solutions for nonlinear systems with gyroscopic terms. Nonlinear Anal. 75, 5756-5764 (2012)

27. Han, Z, Wang, S, Yang, M: Periodic solutions to second order nonautonomous differential systems with gyroscopic forces. Appl. Math. Lett. 24, 1343-1346 (2011)

28. $\mathrm{Li}, \mathrm{X}, \mathrm{Wu}, \mathrm{X}, \mathrm{Wu}, \mathrm{K}$ : On a class of damped vibration problems with super-quadratic potentials. Nonlinear Anal. 72 , $135-142(2010)$

29. Zhang, X: Infinitely many solutions for a class of second-order damped vibration systems. Electron. J. Qual. Theory Differ. Equ. 2013, 15 (2013)

30. Rabinowitz, PH: Minimax Methods in Critical Point Theory with Applications to Differential Equations. CBMS Regional Conf. Ser. in Math., vol. 65. Am. Math. Soc., Providence (1986)

31. Bartolo, P, Benci, V, Fortunato, D: Abstract critical point theorems and applications to some nonlinear problems with strong resonance at infinity. Nonlinear Anal. 7, 241-273 (1983)

32. Luo, Z, Xiao, J, Xu, Y: Subharmonic solutions with prescribed minimal period for some second-order impulsive differential equations. Nonlinear Anal. 75, 2249-2255 (2012)

10.1186/1687-2770-2013-218

Cite this article as: Zhang: Subharmonic solutions for a class of second-order impulsive Lagrangian systems with damped term. Boundary Value Problems 2013, 2013:218 